\title{
Towards a Knowledge-Based Recommendation System for Genomics Visualization
}

\author{
Aditeya Pandey* \\ Northeastern University
}

\author{
Sehi L'Yi \\ Harvard Medical School
}

\author{
Nils Gehlenborg \\ Harvard Medical School
}

\begin{abstract}
Analysis and interpretation of genomics data are the backbones of breakthroughs and discoveries in biomedical research. Visualization tools and techniques play a significant role in the workflow of genomics researchers, and they are regularly employed in the interpretation of genomics data. However, the vast majority of genomics researchers have little or no formal training in data visualization design. Therefore, they require guidance on designing effective visualizations for a given set of data and analysis tasks. In this poster, we present the methodology behind a recommender system for genomics data and our preliminary design of a knowledge-based recommendation system. The system allows genomics researchers to navigate through a selection of visualization options and identify the techniques that meet their preferences and requirements.
\end{abstract}

Index Terms: Human-centered computing-VisualizationVisualization design and evaluation methods

\section{INTRODUCTION}

A rapid growth in the availability of genome and epigenome data has been driving a revolution in how research in biology and medicine is conducted. Visualization plays a critical role in generating knowledge and communicating insights into genomics data in the biomedical research community. Over the years, the biomedical and visualization research communities have published hundreds of tools and visualization techniques to facilitate exploration and analysis of how genomics data are organized [4]. However, through expert interviews and surveys, we found that domain experts lack training in data visualization, thus their choice of visualization tools is often ad hoc and determined through trial and error. Furthermore, many domain experts are unfamiliar with the state of development in genomics visualization, and they may resort to using tools that they have been using in the past or only on the basis of the data formats that they support.

In the visualization community, knowledge-based recommendation systems incorporate design guidelines derived from existing graphical perception studies to support novice users in choosing effective visualization techniques $[3,5]$. However, general purpose visualization recommendation systems may not sufficiently support users in specific domains because the systems do not necessarily cover the design space of domain-specific visualizations and analytical tasks can be different.

In this poster, we present preliminary results on building a recommendation system for genomics visualization. More specifically, we identify six stages for constructing genomics visualizations and suggest how different data features and analytical tasks can affect the recommendation of different visualization designs in those stages (Sec. 3). We also suggest our approach for building a knowledgebased recommendation system for genomics visualization, which can be employed for other domains beyond genomics field (Sec. 2).

\footnotetext{
*e-mail: pandey.ad@northeastern.edu

†e-mail: sehi_lyi@hms.harvard.edu

†e-mail: nils@hms.harvard.com
}

\section{Recommendation Model Creation Methodology}

The first step in building the recommendation system was to identify the data structures, analytical tasks, and visualization designs used in genomics analysis. The required information was gathered from the survey paper by Nusrat et al. [4], where the authors contributed a data, task, and visualization taxonomy for genomics visualization. Overall, we realized that for a domain-specific recommendation system, a taxonomy explicitly defines the input (the data and task) and output specification (visualization) of the model.

Next, we characterized the typical design workflow of a genomics visualization. As shown in Fig. 1B, to create a genomics visualization, a designer needs to make several design decisions like the choice of marks and channels to encode genomics data or how to layout the marks and channels. Our analysis found that design stages are sequential, meaning each step feeds into the next one. For instance, the choice of alignment depends on the choice of layout. Identifying typical design workflow allows us to model our recommendation system in a way that helps genomics researchers to identify components that they are familiar with.

The third step in creating a recommendation model was identification of decision rules that ultimately inform the selection of a visualization. A decision rule can be broken down into an antecedent that represents the data and task constraints of the domain and the consequent, which is the output visualization that effectively supports the data and task requirements. For instance, antecedent:, if the datatype is quantitative and the task is a comparison, consequent:, then the recommendation model should suggest the use of length channel to encode the value [1]. Decision rules that we use for our recommendation models are derived from general visualization graphical, and perception studies [1] and analysis of genomics visualization literature published at visualization conferences [2].

\section{Knowledge-Based Recommendation System}

Conventionally, recommendation systems consist of three main components: (1) inputs, (2) a recommendation model, and (3) recommendation outputs. In this section, we provide an overview of how do we adapt the components of recommendation systems for genomics visualization.

Data and Task Description. We consider both data features and types of analytical tasks as potential input descriptions for our recommendation model as they are pivotal to the design of genomics visualization (Fig. 1A). The data specifications for a genomics visualization inform the recommendation model about the data type of variable that needs to be visualized and other metadata information like the overall volume of the scale of the data. Such information about data can be either provided explicitly by users or collected automatically from the standard file formats used for genomics data. In addition to data-driven recommendations, our system will support task-based recommendations. Typical tasks in a genomics data analysis include comparing two feature values, identifying a correlation between two datasets, and summarizing the entire genomics data distribution [4]. Unlike data descriptions, tasks that users are intended to perform are difficult to infer, which requires task descriptions to be explicitly specified by users.

Recommendation Model. To translate the data and task descriptions into effective genomics visualizations, we propose a sequential 

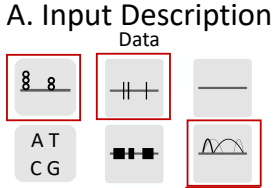

L Lookup

Locate

Browse

Explore
B. Sequential Vis Recommendation

1.Encode 2.Layout 3.Align 4.Arrange 5.View 6.Link
C. Final Output

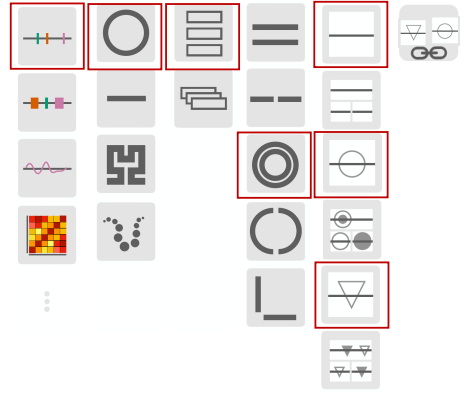

$\longrightarrow$

Recommendation Output Generation

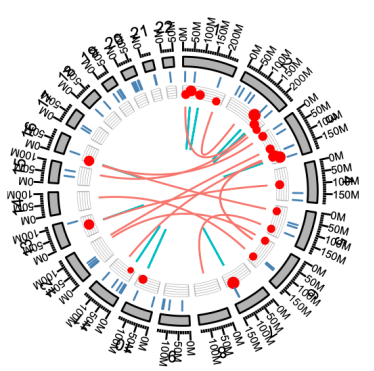

Figure 1: A schematic representation of the three stages of a visualization recommendation system for genomics data. The input visually presents the data and task specification for the system. Based on data and task requirements, the sequential recommendation model identifies suitable visual encoding at each step of the visualization grammar, and the output visualization represents the final deliverable to the user for their analysis.

knowledge-based recommendation model. We use a sequential model to mimic the common workflow of designing genomics visualizations as discussed in Sec. 2. Based on our prior work, we identified the following six main design stages for genomics visualizations (Fig. 1B).

1. Encoding Selection. The first step is the selection of appropriate visual mark or channel to encode genomics data. Based on the results of perception studies (e.g., [5]), our recommendation system suggest a visual mark and a visual channel that are most likely to be effective with the given data type and tasks.

2. Track Layout. After selecting the encoding, individual data points in a genomics dataset are arranged in a layout that preserves their sequential nature (Fig.1B-Layout). In practice, most genomics visualizations show the coordinate systems either linearly or circularly, but there are some alternatives, such as a space-filling layout. The correct layout selection directly affects how the visualization supports tasks such as the comparison of genomics features.

3. Alignment Method. The tracks in genomics visualizations can be parallel aligned to reduce visual clutter, or the information can be superimposed to display an overview rather than showing all individual features (Fig.1B-Align). The selection of alignment often depends on the type of layout users choose in Stage 2. Linear and circular layouts have different visual outputs for the alignment techniques.

4. Track Arrangement. Axes of the same track in the Track Layout can be arranged in different forms (Fig.1B-Arrange). The arrangement of tracks within a design is used to visualize interconnection features and compare two different sequences in comparative genomics. Therefore, the selection of track layout is governed by guidelines that inform us about the effectiveness of a track arrangement to support the connection and comparison analysis tasks [2].

5. View Configuration. A genome sequence can contain billions of nucleotides and analyzing it on different scales, or analyzing more than one region of interest at a time can be of great value. The view configuration step of the model gathers the user's high-level analytical requirements and describes the scale, foci, and views to use to visualize the genomics data (Fig.1B-View).

6. Linked Views. The last step in the design of a genomics visualization is the configuration of linked views. Linked views are usually defined between the views recommended at the View Configuration stage. This recommendation step aims to identify the interactive features to implement between the views to allow simultaneous navigation and exploration, playing a vital role in the comparative analysis of genomes.

Choosing a sequential model was a natural choice because of the incremental and dependent nature of genomics visualizations. At each stage, the recommendation model acts on a set of user-defined or implicitly determined inputs. For instance, during the Encoding Se- lection, the input consists of the feature data type and tasks. Then the decision rules at the stage determine the most appropriate visual output corresponding to the stage. The incremental model allows users to validate the visualization at the early stages of design and learn about the design practices recommended in the visualization literature.

Recommendation Output. Existing visualization tools support limited encoding, tasks, and features [4]. Therefore, we will implement a JavaScript library, a web-based framework for generating custom genomic visualizations. It will enable the recommendation model to easily create and deploy custom interactive multi-scale visualizations as a web-based tool. A customized recommended visualization output is shown in Fig. 1C.

\section{Conclusion AND Future Work}

This poster presents a recommendation system design that aims to support genomics researchers with limited visualization experience to generate valid and meaningful genomics visualizations. Our preliminary model design methodology highlights the importance of surveying data and task requirements to create a domain-specific recommendation system. Furthermore, the proposed recommendation system uses a sequential recommendation model, that allows researchers to develop the visualization incrementally and adds transparency to the visualization recommendation process. We are currently designing the user interface of the recommendation tool that will be used for genomics researchers. Additionally, we are working on broader research questions such as the method of task input for a visualization recommendation. We also aim to validate the proposed model with more domain experts. Overall, we believe our proposed method will enable the development of a reliable and suitable visualization recommendation tool for genomics researchers.

\section{REFERENCES}

[1] W. S. Cleveland and R. McGill. Graphical perception: Theory, experimentation, and application to the development of graphical methods. Journal of the American Statistical Association.

[2] M. Meyer, T. Munzner, and H. Pfister. Mizbee: A multiscale synteny browser. IEEE TVCG. doi: 10.1109/TVCG.2009.167

[3] D. Moritz, C. Wang, G. L. Nelson, H. Lin, A. M. Smith, B. Howe, and J. Heer. Formalizing visualization design knowledge as constraints: Actionable and extensible models in draco. IEEE TVCG. doi: 10.1109/ TVCG.2018.2865240

[4] S. Nusrat, T. Harbig, and N. Gehlenborg. Tasks, techniques, and tools for genomic data visualization. CGF. doi: 10.1111/cgf. 13727

[5] B. Saket, D. Moritz, H. Lin, V. Dibia, C. Demiralp, and J. Heer. Beyond heuristics: Learning visualization design. arXiv:1807.06641. 